\title{
Development of T - joints Automatic Welding Equipment for Jacket Based on Saddle - shaped Space Trajectory
}

\author{
Mingzhu Liu ${ }^{1, a}$, Kekuan Wang ${ }^{1, b}$, Bin Long ${ }^{1, c}$ and Tianli Zhang ${ }^{1, d}$ \\ ${ }^{1}$ CNPC Research Institute of Engineering Technology Tianjin, P. R. China \\ aliumz@cnpc.com.cn, ${ }^{b}$ wangkekuan@cnpc.com.cn, ${ }^{9}$ longb@cnpc.com.cn, ${ }^{\mathrm{d}}$ zhangtl@cnpc.com.cn
}

Keywords: jacket, T-joints, automatic welding.

Abstract. Through the saddle intersection curve analysis, the mathematic model of the saddle-shaped space trajectory was established by matrix equation and interpolation method. For the special saddle-shaped welds of T-joints the automatic welding device is designed for automatic welding of jacketed T-joints, which consists of a traveling device with the cone-wheel elastic positioning mode, a track device with elevation engagement and a swing mechanism with high-precision linear modules. The equipment used in WHPE platform construction of Suizhong 36-1 project, and achieved good results.

\section{Introduction}

In recent years, China has made great progress in many aspects such as offshore platforms, oil and gas delivery, submarine pipelines and deep diving technology. With the continuous increase of energy demand, the development of offshore oil and gas resources will enter a new historical period and inevitably promote the rapid increase in the number of platform construction. The jacket is the steel structure foundation of the offshore oil platform, and the welding of pile pipes is an indispensable part in the installation and construction of the jacket ${ }^{[1]}$. T-joints in the manufacture of jackets, as a result of more nodes, groove depth, and involves all-position welding, not only weld metal welding volume, and the difficulty of operation. At present, most of the construction of T-joints of jackets at home and abroad using manual arc welding method. As the jacket wall thickness and a large amount of welding, the use of manual welding efficiency is low and the welding quality is difficult to maintain stability ${ }^{[2]}$. Therefore, the use of advanced jacket $\mathrm{T}$-joints automatic welding equipment has a very important economic significance, which improved the welding efficiency and shortened the construction period.

\section{T-joints space trajectory planning}

T-type pipe joints are a common form of assembly in the installation of piping structural parts, which generally used manual welding due to technical conditions. In order to introduce the automatic welding technology into the T-joints welding work, the space trajectory planning of the jacket should be studied firstly for controlling the robot weldling automatically. T-junction is different with the general two-dimensional weld, which is a line of intersection. As the intersection line is a spatial curve which is still in a different angle position, the moving trajectory of the weld torch is more complex for automatic welding. It involves multi-dimensional, multi-degree of freedom control problems, but also involves the welding process of different welding positions ${ }^{[3]}$. As the T-shape welds change in three-dimensional space, the spatial tangent and the normal plane of the path are changed. During the welding process, the attitude of the weld torch needs to be planned to guide the automatic welding of the robot according to the tangent of each point, the normal plane direction and the dimension of the pipe joints. 


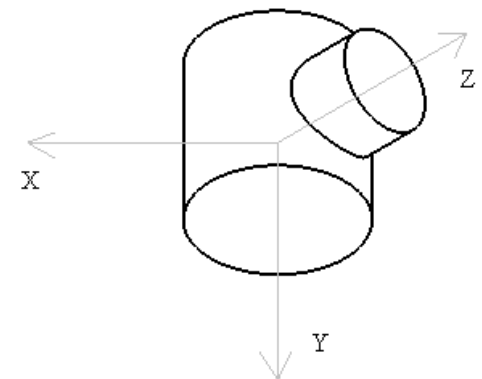

Fig.1 Workpiece model

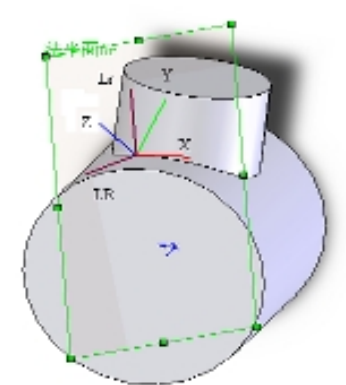

Fig.2 Weld coordinate system

The workpiece model is shown in Figure 1, where the cylindrical intersection curve equation can be obtained:

$$
\left\{\begin{array}{l}
x^{2}+y^{2}=r_{2}^{2} \\
z^{2}+x^{2}=r_{1}^{2}
\end{array}\right.
$$

$n_{x}, n_{y}, n_{z}, o_{x}, o_{y}, o_{z}, a_{x} 、 a_{y}, a_{z}$ can be used to describe the parameters of the spatial attitude of the weld torch. The homogeneous coordinate matrix can be obtained ${ }^{[4]}$ :

$$
\left[\begin{array}{lll}
n_{x} & o_{x} & a_{x} \\
n_{y} & o_{y} & a_{y} \\
n_{z} & o_{z} & a_{z}
\end{array}\right]
$$

Assuming that the tangent direction of each point on the curve is the moving direction of the weld torch, Since the tangential direction has two directions, supposing that the rotation of the tangent about the positive $\mathrm{z}$-axis is positive.The spatial curve equation is obtained:

$$
\left\{\begin{array}{l}
F(x, y, z)=0 \\
G(x, y, z)=0
\end{array}\right.
$$

where the tangent equation can be obtained:

$$
\frac{x-x_{0}}{\left|\begin{array}{ll}
F_{y} & F_{z} \\
G_{y} & G_{z}
\end{array}\right|_{0}}=\frac{y-y_{0}}{\left|\begin{array}{cc}
F_{z} & F_{x} \\
G_{z} & G_{x}
\end{array}\right|_{0}}=\frac{z-z_{0}}{\left|\begin{array}{cc}
F_{x} & F_{y} \\
G_{x} & G_{y}
\end{array}\right|_{0}}
$$

where the normal plane equation can be obtained:

$$
\left|\begin{array}{ll}
F_{y} & F_{z} \\
G_{y} & G_{z}
\end{array}\right|_{0}\left(x-x_{0}\right)+\left|\begin{array}{cc}
F_{z} & F_{x} \\
G_{z} & G_{x}
\end{array}\right|_{0}\left(y-y_{0}\right)+\left|\begin{array}{cc}
F_{x} & F_{y} \\
G_{x} & G_{y}
\end{array}\right|_{0}\left(z-z_{0}\right)=0
$$

According to equation (1), the tangent equation of point $(x(i), y(i), z(i))$ can be obtained:

$$
\frac{x-x(i)}{\left|\begin{array}{cc}
2 y(i) & 0 \\
0 & 2 z(i)
\end{array}\right|}=\frac{y-y(i)}{\left|\begin{array}{cc}
0 & 2 x(i) \\
2 z(i) & 2 x(i)
\end{array}\right|}=\frac{z-z(i)}{\left|\begin{array}{cc}
2 x(i) & 2 y(i) \\
2 x(i) & 0
\end{array}\right|}
$$

And the tangent vector is $\left(\mathrm{n}_{\mathrm{x}}, \mathrm{n}_{\mathrm{y}}, \mathrm{n}_{\mathrm{z}}\right)=(-\mathrm{y}(\mathrm{i}) \mathrm{z}(\mathrm{i}), \mathrm{x}(\mathrm{i}) \mathrm{z}(\mathrm{i}), \mathrm{x}(\mathrm{i}) \mathrm{y}(\mathrm{i}))$, the direction of which is also the $\mathrm{X}$-axis direction of the weld torch coordinate system. So that the normal equation of the plane $\mathrm{Ar}$ which passing through this point can be obtained:

$$
\left|\begin{array}{cc}
2 y(i) & 0 \\
0 & 2 z(i)
\end{array}\right|(x-x(i))+\left|\begin{array}{cc}
0 & 2 x(i) \\
2 z(i) & 2 x(i)
\end{array}\right|(y-y(i))+\left|\begin{array}{cc}
2 x(i) & 2 y(i) \\
2 x(i) & 0
\end{array}\right|(z-z(i))=0
$$


The weld coordinate system is shown in Figure 2. Define LR as the tangent to the point which passing through the curve formed by the intersection of the normal plane Ar and the curve surface of the main pipe. So that the intersection curve equation is obtained:

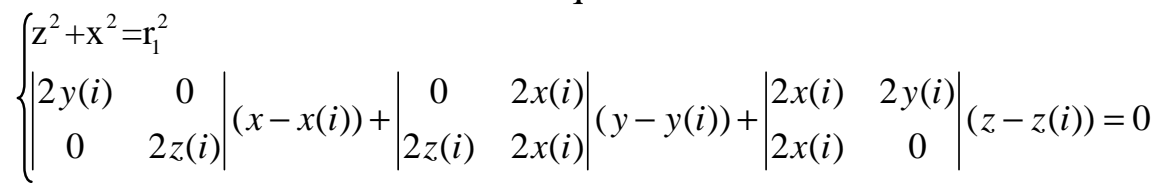

The tangent of this point is:

$$
\frac{x-x(i)}{\left|\begin{array}{cc}
0 & 2 z(i) \\
-x(i) z(i) & -y(i) x(i)
\end{array}\right|}=\frac{y-y(i)}{\left|\begin{array}{cc}
2 z(i) & 2 x(i) \\
-y(i) x(i) & y(i) z(i)
\end{array}\right|}=\frac{z-z(i)}{\left|\begin{array}{cc}
2 x(i) & 0 \\
y(i) z(i) & -x(i) z(i)
\end{array}\right|}
$$

Define $\mathrm{Lr}$ as the tangent to the point which passing through the curve formed by the intersection of the normal plane Ar and the curve surface of the branch pipe. So that the intersection curve equation is obtained:

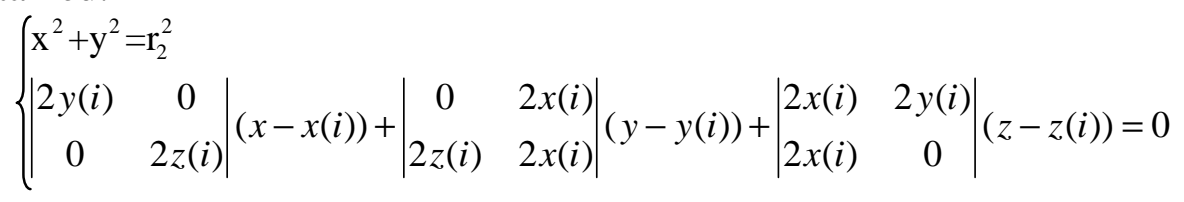

The tangent of this point is:

$$
\frac{x-x(i)}{\left|\begin{array}{cc}
2 y(i) & 0 \\
-x(i) z(i) & -y(i) x(i)
\end{array}\right|}=\frac{y-y(i)}{\left|\begin{array}{cc}
0 & 2 x(i) \\
-y(i) x(i) & y(i) z(i)
\end{array}\right|}=\frac{z-z(i)}{\left|\begin{array}{cc}
2 x(i) & 2 y(i) \\
y(i) z(i) & -x(i) z(i)
\end{array}\right|}
$$

The weld torch posture of path points required in the century coordinate system is shown in Figure 3 , the matrix of path points is obtained:

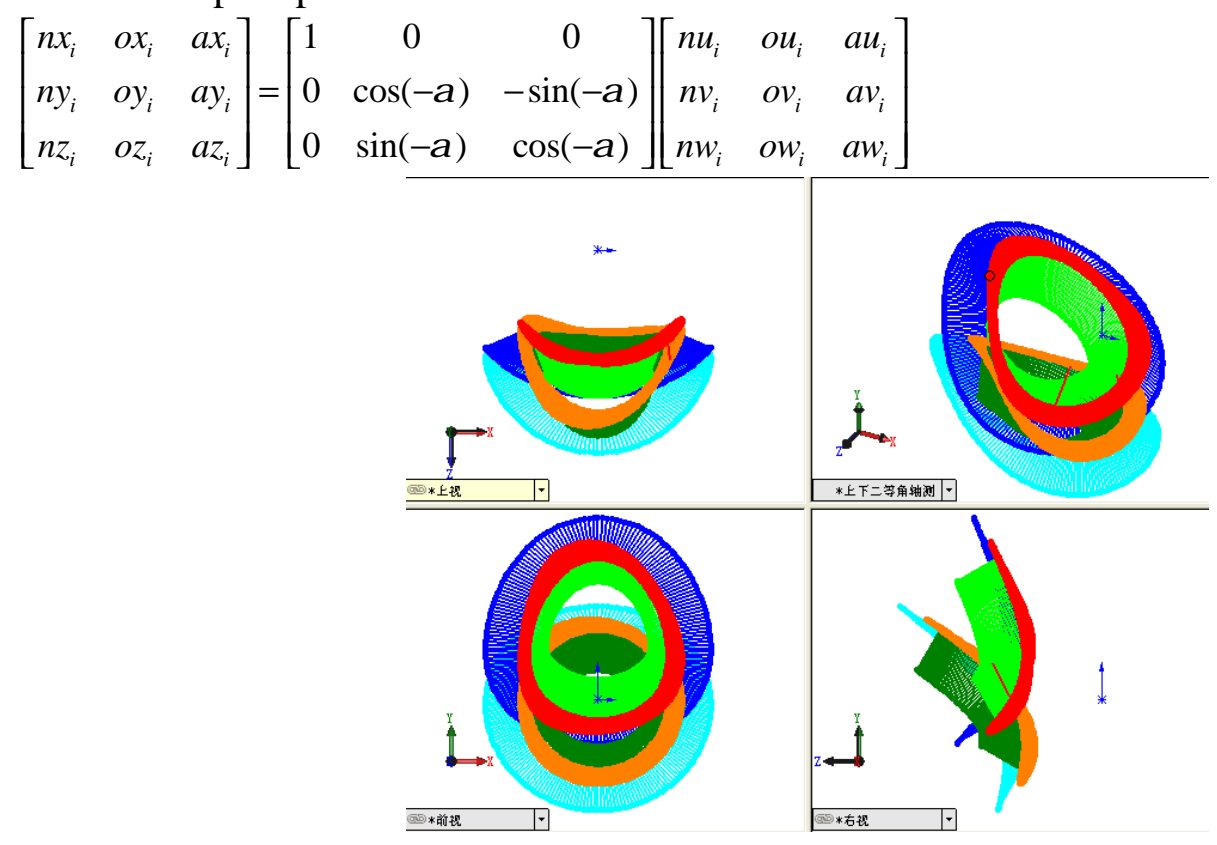

Fig.3 The planning result of T-shape weld path

\section{Mechanical structure of $T$-joints automatic welding equipment for jacket}

The mechanical structure of T-joints automatic welding equipment for jacket is shown in Figure 4. It is mainly composed of the traveling device, the swing device and the the track device and other 
components. The traveling device is designed by the cone-wheel elastic positioning mode, which uses the gear transmission to realize the movement along the circumferential direction of the riser. The track device is designed by the conical structure on both sides, which achieves locking through the eccentric locking mechanism. The swinging device is designed by the structure of the single-arm robot and the linear module, which achieves the reciprocation and the wire extension control movement of the weld torch. At the same time it is designed by the shaft structure to achieves the weld angle control and the weld torch swing function.
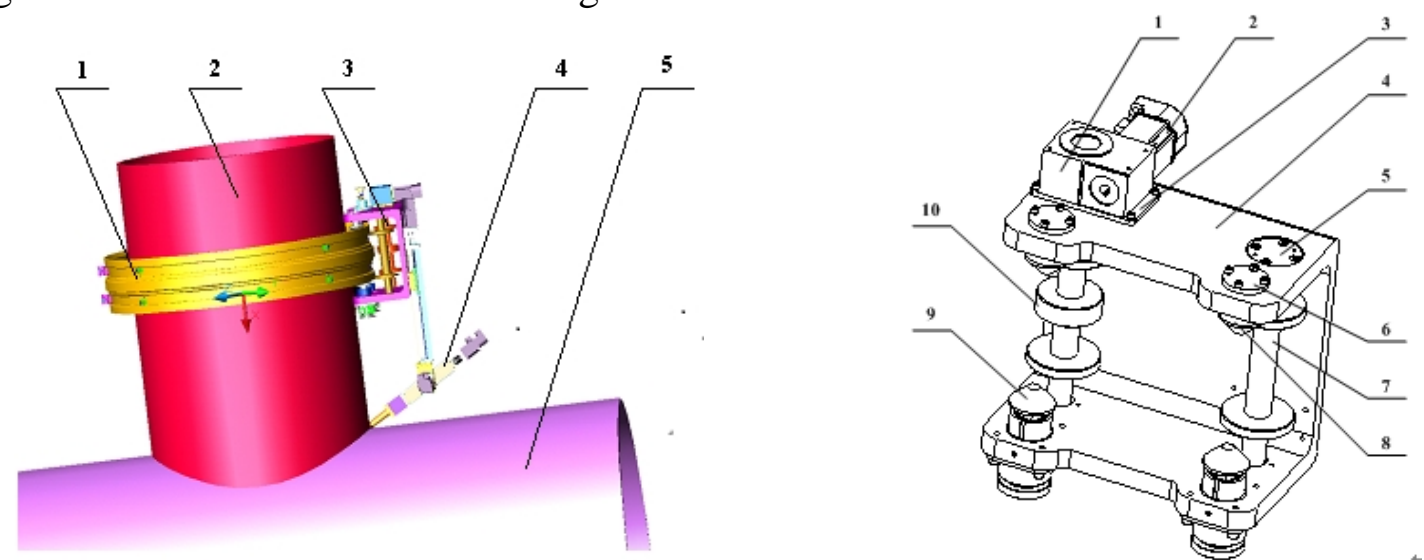

1.Track device 2.Riser 3.Traveling device

1.Worm reducer 2. Traveling motor 3. Connection plate

4. Swing device 5. Main pipe

Fig.4 Structure diagram of the equipment
4. Frame 5. Bearing cover 6. Cone-wheel cover

7. Slave axis 8. Fixed cone-wheel

9. Movable cone-wheel 10. Gear shaft

Traveling device. The traveling device is shown in Figure 5, it adopts the friction driving mode, that is, the transmission mode with the cone-wheel which has the elastic adjusting and locating function and the conical track. Finally, it can be completing the circumferential movement of the automatic welding equipment along the circumference of the riser. The device adopts the way that the tooth surface on the track ring gear is distributed along the circumferential direction, which is different from the side rack meshing transmission in the pipeline all-position automatic welding. It can improve the transmission accuracy, the weld tracking accuracy and the crawling stability. At the same time, by means of fixed cone- wheel and the conical track positioning, the traveling device can be quickly installed on track. The positioning mode makes the device can be applied to a variety of different size diameter of the track, which greatly improve the applicability of the device. The traveling device is clamped on the track by adjusting cone-wheels in which a biaxial structure between two shafts using disc spring elastic connection is designed. It can be clamped on the track quickly through adjusting the position of the movable cone-wheel. At the same time, It can automatically adjust the tightness of the clamping track by the elastic positioning function of movable cone-wheels during the crawling process, and prevent the machining error and deformation of the track from being stuck in the crawling process.

Swing device. The traveling device is shown in Figure 6, it can realize the equipment along the riser axis reciprocating motion, the weld torch control movement, the wire extension control movement, the weld angle control and weld torch swing function. The output shaft of the lifting motor is connected to the screw guide of the single-arm robot, which is driven by the lifting motor to achieve the reciprocating motion of the single arm robot. The slider of the single arm robot is fixed on the frame of the traveling device, it realizes the connection between the swing device and the traveling device. When the lifting motor drives screw guide, the single-arm robot will be reciprocating. It can realize the weld torch the reciprocating motion along the axial direction of the riser. The swing 
mechanism is connected with the linear module, by which the angle regulation and the micro swing of the weld torch is realized.

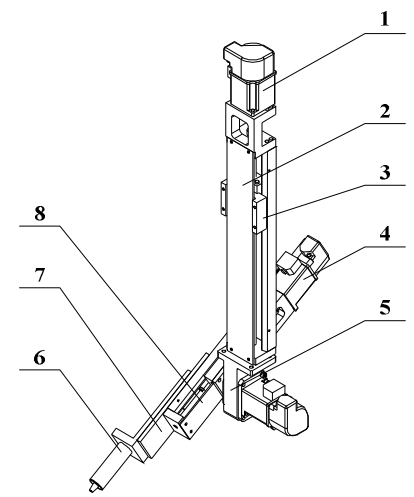

1. Lifting motor 2. Single-arm robot 3. Slider

4. Wire extension motor 5. Swing mechanism 6. Welding mechanism 7. Welding connection frame 8. Linear module

Fig.6 Structure diagram of the swing device

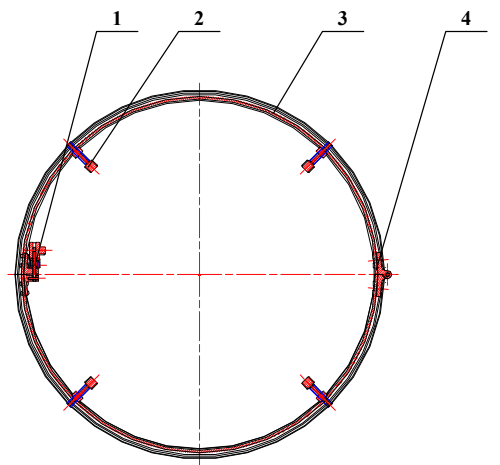

1. Eccentric wheel lock 2. Supporting frame 3. Track 4. Hinge

Fig.7 Structure diagram of the track

Track device. According to the special cone-wheel elastic positioning mode, the corresponding track is also used to adapt the special cone-wheel positioning mode. Both ends of the track are cone, which is convenient for positioning. The outer cylindrical surface of the maximum diameter of the track is a gear ring, which is arranged along the circumferential direction, and can be engaged with the gear on the gear shaft of the traveling device to ensure the running stability. The structure of the track as shown in Figure 7, it is composed of an eccentric wheel lock, supporting frame, track and hinge. The eccentric wheel lock can be used for positioning and locking, and the supporting frame can be supported on the jacket. The track is two bivalve structure, which can be opened and closed through the hinge. To achieve the rapid installation of the automatic welding equipment, the track should be as light as possible, at the same time must be able to tighten the card, in order to ensure the safety of equipment in the construction process.

\section{Control system of $\mathrm{T}$-joints automatic welding equipment for jacket}

The control system adopts advanced digital control, the industrial computer plugs the PCI interface of the DMC9940 motion control card, and the motion control card is connected to ICM-1900ID interface board by special cables. The driving circuit of the interface board is connected with the motor driver, and the digital control of each axis is carried out. The hand-box of the automatic welding equipment is designed and produced by the input of the digital interface of DMC 9940 and the input of the analog interface. The welder can set welding parameters by the hand-box, and the micro adjustment of the track of the traveling device in the welding process is realized. The principle of control system is shown in figure 8 . 


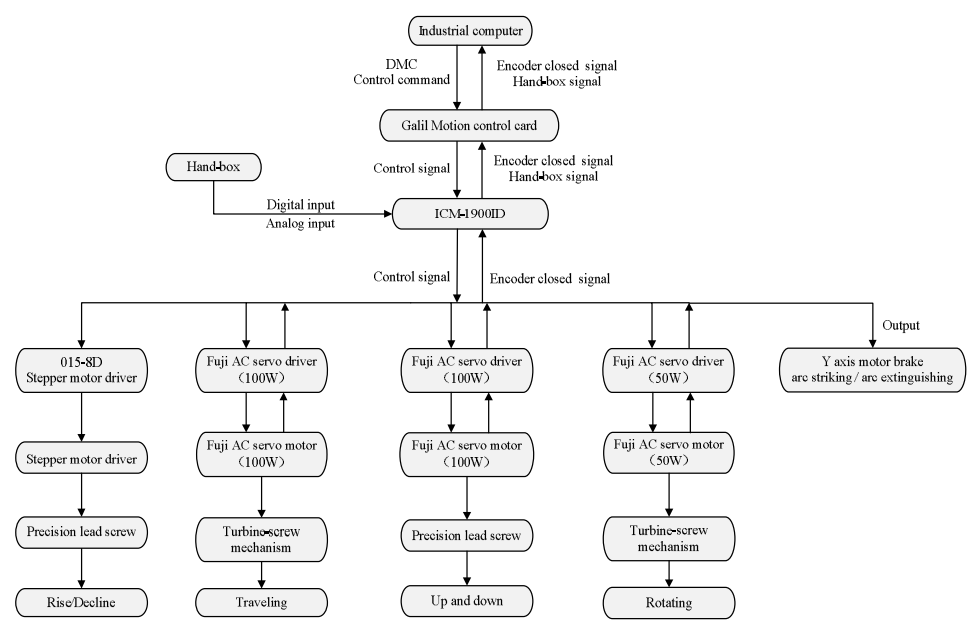

Fig. 8 Principle diagram of control system

\section{Engineering application}

The T-joints automatic welding equipment is applied to the construction of the WHPE platform in Suizhong 36-1 project, which was constructed by the CNPC Offshore Engineering Company Limited in August 2010.

The jacket is welded by using the T-joints automatic welding equipment, and the diameter of the main pipe is 1115 millimeter, the diameter of the riser is 610 millimeter. In the welding progress the welding power source adopts Miller Invision 456P type source, the wire feeder adopts X-Treme 12VS type, the welding wire adopts Lincoln self-protective flux cored wire, and it adopts self-shielded welding method. The welding process test is shown in figure 9, and the weld formation diagram is shown in figure 10. This project adopts semi-automatic welding and sealing, completed a total of five crossing of the T-joints, crossing each weld layer 22-28. The application shows that the device can automatically identify the saddle welding trajectory, stable operation, good controllability, reasonable process parameters, weld appearance, to meet the requirement of welding T-joints of the jacket. It is two times more efficient than manual welding, and can reduce the labor intensity of welders. The qualification rate of the first pass of weld is above ninety-eight percent, which meets the requirements of engineering acceptance specifications.

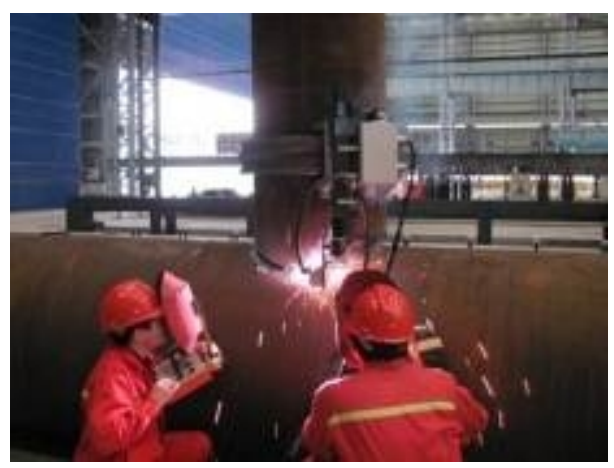

Fig.9 Welding process test

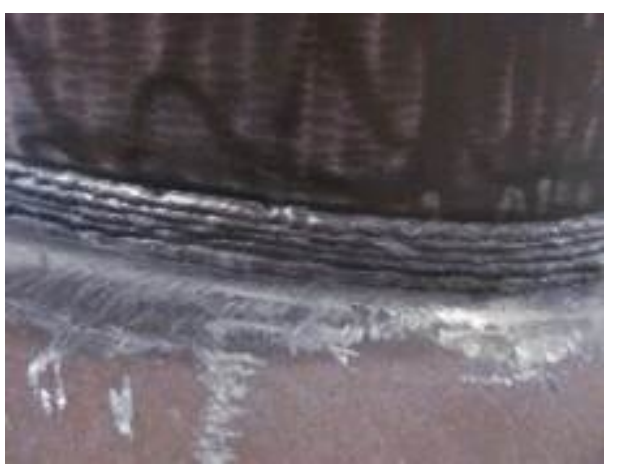

Fig.10 Weld formation diagram

\section{Conclusions}

In order to improve the quality of pipeline welding and welding productivity, reduce labor intensity, ensure safe and stable operation of the offshore platform, it is a top priority to develop the T-joints 
automatic welding equipment for jacket, which can be used in field work. The development of the equipment has a broad application prospects and promotion prospects, and it will create a high economic efficiency and significant social benefits.

\section{References}

[1] Li Qingping: The challenge of offshore deepwater oil and gas development in China. China offshore oil and gas. 2006,18 (2): 120P

[2] Giovanni Corbetta \& David S. Cox: Deepwater Tie-ins of Rigid Lines: Horizontal Spools or Vertical Jumpers. 1999 Offshore Europe Conference. 1999:2,3P

[3] Duan Tiequn, Meng Fanrong and Yu Dan:Motion simulation of the saddle shaped automatic welding machine based on ADAMS. Welding production application. 2008,11 (1): $64 \mathrm{P}$

[4] Zhang Mu, Li Liangyu and Wang Tianqi: Offline programming system of offshore platform jacket arc welding robot based on SolidWorks. Journal of Shanghai Jiao Tong University. 2008,42 (1): $10 \mathrm{P}$ 